\title{
El migragrama: una propuesta metodológica para el estudio de las remesas económicas
}

\author{
JESÚS SANZ ABAD \\ Universidad Complutense de Madrid \\ jsanzabad@hotmail.com
}

Recibido: 13.06 .2009

Aceptado: 24.02 .2010

\section{EL MARCO TEÓRICO: LA PERSPECTIVA TRANSNACIONAL}

Desde los años setenta, se ha producido un consenso teórico en las ciencias sociales sobre la necesidad de considerar simultáneamente los procesos económicos, políticos, demográficos y culturales a escala global y local, a la vez que se destaca cada vez más el impacto que ejerce el sistema mundial sobre las realidades locales y la interconexión e interdependencia cuantitativa y cualitativa que existe entre las distintas regiones del mundo.

Estos hechos se han asociado a una nueva fase del sistema capitalista caracterizada por la internacionalización de la producción, la concentración del capital, nuevas formas de acumulación flexible y un declive en la importancia del Estado-nación en la gestión y la planificación económica y política. Al mismo tiempo, ligados a los cambios en los modos de producción se han producido importantes cambios sociales y culturales fruto de las innovaciones tecnológicas y la difusión de las mismas que se ha producido a partir de estos años.

Con ello, los avances tecnológicos producidos en los últimos años en campos como la comunicación, los transportes o los flujos de información han posibilitado la contracción de las categorías espacio-temporales y han permitido la progresiva interconexión entre culturas, gentes y lugares, así como la interconexión y la (re)construcción de espacios de intercambio y de intercomunicación.

Las posibilidades que se abren a partir de estos cambios han sido aprovechadas entre otros actores por los migrantes, quienes recurriendo a diferentes instrumentos relativizadores de la distancia, han intensificado los vínculos y contactos con sus países de origen a través de diferentes modos, a la vez que han modificado sustancialmente conceptos como el de «retorno».

La fluidez en las comunicaciones y las constantes relaciones realizadas entre el país de origen y el país de destino u otros, también están en el origen del tér- 
mino transnacionalismo, un termino con el que se pretende definir los procesos a través de los cuales los migrantes forjan y mantienen constantemente relaciones sociales que vinculan sus sociedades de origen y de destino. Este concepto ha alcanzado una gran popularidad en los últimos años debido al aumento espectacular de las prácticas transnacionales en múltiples ámbitos (ideológico-culturales, políticos, religiosos, económicos, etc.).

Sin embargo, en el caso del transnacionalismo la novedad no estaría tanto en que se trate de un fenómeno nuevo, sino en la existencia de una nueva perspectiva teórica emergente (la perspectiva transnacional) que pretende aportar una nueva visión del fenómeno migratorio a partir de unos presupuestos teóricos novedosos.

Esta perspectiva transnacional se ha convertido en los últimos años en el paradigma dominante dentro de los estudios sobre migraciones, con una visión en la que las migraciones son analizadas como procesos complejos articuladores de espacios geopolíticos y culturales. Esta perspectiva supone una importante renovación teórica dentro de los estudios sobre migraciones al romper con el nacionalismo metodológico (la consideración del Estado-nación como el marco natural para el estudio de los procesos migratorios), con el individualismo metodológico como punto de partida para el análisis de los flujos migratorios, y con el énfasis puesto en la asimilación como eje de las estrategias de adaptación de los migrantes.

Igualmente, desde esta perspectiva también se destaca la necesidad de examinar los contextos de partida de los migrantes para entender las lógicas que existen en la migración, frente a primar el análisis únicamente en la sociedad receptora, recogiendo lo señalado previamente por autores como Sayad (1999), para el que la inmigración y la emigración son dos caras indivisibles de una misma realidad que no pueden ser comprendidas cuando una de las dos partes del binomio está ausente. Con ello, la migración es considerada como un «hecho social total» que para ser entendido no debe realizar separaciones rígidas entre el «antes» y el «después» de la migración, o entre el país de origen y el país de llegada, y donde las sociedades de emisión y de recepción se ven como un campo continuo y único de análisis.

\section{DESAFÍOS METODOLÓGICOS DENTRO DE LA PERSPECTIVA TRANSNACIONAL}

La necesidad de reconocer las interconexiones e interdependencias que se producen en una multiplicidad de lugares como propone la perspectiva transnacional, supone un desafío que requiere de una pluralidad de técnicas, metodologías y marcos de referencia teóricos-conceptuales bajo los cuales pensar la realidad, y trascender los esquemas binarios clásicos de la investigación sobre migración.

Con el fin de abordar esta cuestión, dentro de la perspectiva transnacional cada vez se han hecho más frecuentes las investigaciones basadas en un diseño 
y concepción multilocal, a la vez que algunos autores como Levitt y Glick Schiller (2004) han visto en la etnografía una herramienta especialmente adecuada en el estudio de estas interdependencias, por sus posibilidades para analizar cómo las personas interaccionan dentro de un espacio y más allá de sus fronteras, y cómo actúan de forma que se refuerzan o contradicen sus valores (Solé, Parella y Cavalcanti, 2007).

Una de las propuestas más interesantes que a nivel metodológico ha ido en esta dirección ha sido la denominada por Marcus (1995) etnografía multisituada o multi-local. En un contexto marcado por el multilocalismo, la desterritorialización y los flujos constantes de personas, de recursos y de información, la etnografía multisituada se distingue por poseer un objeto de estudio que no puede ser explicado etnográficamente si se realiza trabajo de campo intensivo en un solo lugar; sino que se ha de aprehender por medio de una «etnografía móvil» que se desenvuelve en múltiples lugares con el fin de examinar la circulación de los significados culturales, los objetos o las identidades en un tiempo-espacio difuso, aun asumiendo que el producto de conocimiento obtenido variará en intensidad y calidad en los diferentes lugares abordados.

Al mismo tiempo, la creciente disociación geográfica existente entre el lugar de estudio donde se realiza la investigación y el objeto de estudio a abordar, así como entre las diferencias existentes entre la escala de observación micro en la que el etnógrafo desarrolla su trabajo y el marco de análisis mucho más amplio con objetos de estudio separados geográficamente, supone un problema epistemológico al que la etnografía debe hacer frente. En el caso de las migraciones, como señala Appadurai, «en la medida en que los grupos migran, se reagrupan en nuevos lugares, reconstruyen sus historias y reconfiguran sus proyectos étnicos, lo etno de la etnografía adquiere una calidad resbaladiza y no localizada, ante lo cual tendrán que responder las prácticas descriptivas de la antropología», debido a que el estudio de los grupos concretos «dejaron de estar firmemente amarrados a un territorio y circunscriptos [sic] a ciertos límites espaciales» (Appadurai, 1996 (2001): 63).

En este contexto general, se hace necesario repensar los anclajes y nociones con los que realizar la producción de conocimiento, a la vez que se hace necesario prestar más atención a los aspectos dinámicos y contextuales de la realidad social frente a otros aspectos estáticos.

Dentro de esta primacía de los aspectos relacionales, sin duda el concepto que ha cobrado más fuerza en el estudio de las migraciones ha sido el concepto de red, por su capacidad para comparar las experiencias de quienes emigran con las personas que están indirectamente influidos por ideas, objetivos e información que fluyen a través de los flujos migratorios, así como por su capacidad para analizar la circulación de trabajo, capital, bienes, servicios, información e ideologías entre las comunidades que envían migrantes y las que los reciben.

Por otro lado, al utilizar el concepto de red social dentro de la perspectiva transnacional hay que diferenciar al menos entre dos acepciones del mismo: 
- El concepto de red como metáfora o estrategia heurística. Un concepto con el que se alude a la posibilidad de extraer de un sistema más amplio y con propósitos analíticos un conjunto de relaciones sociales.

- El concepto de red como técnica de investigación basada en la representación visual de la realidad a través de una representación gráfica realizada preferentemente con líneas y nodos.

Sin embargo, si bien el concepto de red social ha tenido bastante desarrollo dentro de los estudios sobre migraciones, existe en torno a este concepto un amplio espacio todavía por cubrir entre las formulaciones teóricas realizadas, la escasez de propuestas metodológicas y de técnicas e instrumentos de investigación existentes, y los contextos de estudio a abordar, como se mostrará más adelante con el caso concreto del migragrama.

\section{UNA PANORÁMICA GENERAL DE LOS ESTUDIOS SOBRE REMESAS}

El análisis de los envíos de remesas es uno de los temas que más interés está suscitando como consecuencia de su importancia a nivel mundial, y de su fuerte crecimiento en los últimos años.

En términos generales, dentro de los trabajos relacionados con esta temática se pueden distinguir varias líneas de investigación que han sido ampliamente trabajadas.

- Una primera línea que parte de un enfoque macroeconómico y está dominada por los datos estadísticos, busca determinar la cuantía concreta de las remesas enviadas y/o recepcionadas en un determinado país o región, así como analizar los efectos que éstas tienen a nivel macroeconómico. Algunos de los aspectos que se han debatido en este ámbito han sido los efectos que las remesas tienen en la balanza de pagos y su evolución en relación con otras partidas de la misma balanza, su impacto en la reducción de la pobreza y en una mayor o menor desigualdad social, y la relación entre la recepción de remesas y el aumento de las importaciones de bienes suntuarios.

- Una segunda línea de investigación, que en ocasiones llega a converger con la anterior, busca caracterizar la finalidad de las remesas, el perfil de migrantes y receptores, así como la cuantía, periodicidad y estructura de gasto de éstas ${ }^{1}$.

${ }^{1}$ Algunos trabajos que irían en esta dirección serían, por ejemplo los realizados por Garay y Rodríguez (2005); Ponce, Olivié y Onofa (2008), o las diferentes investigaciones realizadas por la CEPAL en América Latina citadas en Martínez Pizarro (2005). 
La mayor parte de estos trabajos se basan en encuestas de hogares que, sin embargo, en ocasiones presentan ciertos inconvenientes: con cierta frecuencia estos trabajos se centran exclusivamente en la estructura de gasto de los receptores de remesas sin ofrecer una perspectiva comparada entre receptores y no receptores del lugar de estudio, con el consiguiente riesgo de reificación de toda explicación de los resultados obtenidos en torno a la recepción de remesas. Igualmente, si bien la información que aportan estos trabajos es valiosa y útil, en ocasiones éstos incurren en cierto economicismo, ya que al centrarse exclusivamente en el perfil que presentan los receptores y emisores de remesas individualizan este fenómeno y descontextualizan el marco social en el que se producen los envíos de dinero.

- Una tercera línea de investigación, aunque minoritaria y más desconocida, ha ido destinada a señalar algunos aspectos sociales y simbólicos que existen en el uso de las remesas más allá de la lógica económica.

Dentro de esta línea, además de los trabajos que han intentado analizar los efectos de las denominadas remesas sociales ${ }^{2}$ (Levitt, 1996; 2001), diferentes autores han señalado algunos efectos sociales que las remesas presentan en los contextos de origen. Entre otros aspectos se ha destacado la relación entre la recepción de remesas y la alteración de las jerarquías tradicionales (Wamsley, 2001); la vinculación en algunos contextos entre el incremento de dinero circulante procedente de las remesas y el debilitamiento de sistemas de relaciones de intercambio de trabajo y de otras actividades que favorecen la cohesión social (Wamsley,, 2001; Suarez (2007a); el papel que las remesas juegan en el mantenimiento de vínculos duraderos entre los migrantes y sus familiares (Herrera, 2004); las obligaciones morales contraídas en el grupo doméstico en torno a su utilización, o los cambios que se pueden producir en los roles de género en relación con el control de las remesas y los procesos de empoderamiento (García y Palenwonsky, 2006).

A estos aspectos sociales, hay que sumar el simbolismo existente en torno a las remesas (Moctezuma, 2004), así como los efectos que la recepción de remesas tiene en los imaginarios colectivos de las poblaciones locales, al crear nuevos marcadores de status ligados al consumo de bienes.

Con todo ello, se puede afirmar que la inmensa mayoría de los trabajos sobre remesas realizados se centran únicamente en la vertiente económica, ignorando

${ }^{2}$ Levitt define las remesas sociales como «las transferencias culturales instigadas por la migración en aspectos de desarrollo» (1996:2-3), agrupando bajo este término al «conjunto de estructuras normativas y sistemas de prácticas que eran interiorizados por los migrantes durante su experiencia migratoria y que posteriormente eran puestos en práctica en sus comunidades de origen» (Levitt, 2001, traducción del autor).

EMPIRIA. Revista de Metodología de Ciencias Sociales. N. . 19, enero-junio, 2010, pp. 207-232. ISSN: $1139-5737$ 
o relegando a un segundo plano las dimensiones sociales de éstas. Se invisibilizan de este modo algunas dimensiones sociales existentes en torno a las remesas, así como otros aspectos ligados a la economía política del hogar, las relaciones de poder existentes dentro del grupo doméstico, o las estrategias económicas de los migrantes.

Al mismo tiempo, las contribuciones que se han realizado sobre las dimensiones sociales que rodean a éstas, se han basado mayoritariamente en las técnicas de investigación más utilizadas en la etnografía como la entrevista o la observación participante. Con ello, se manifiesta una escasez de propuestas metodológicas y de técnicas de investigación que aporten nuevos enfoques y perspectivas sobre este objeto de estudio.

Surge de este modo, toda una línea de investigación en la que es necesario profundizar y en la cual se enmarca la propuesta metodológica que a continuación se presenta.

\section{UNA PROPUESTA METODOLÓGICA PARA EL ANÁLISIS DE LAS ESTRATEGIAS ECONÓMICAS DE LA MIGRACIÓN: EL MIGRAGRAMA}

Desde el punto de vista metodológico, ¿cómo se puede abordar el estudio de un objeto de estudio tan móvil y resbaladizo como son las remesas? ¿A qué técnicas de investigación que reconozcan las interconexiones entre los diferentes espacios geográficos ocupados por los migrantes y sus familiares se puede acudir para intentar captar un objeto de estudio de estas características? Estas eran algunas de las preguntas que me hacía a la hora de plantear el diseño metodológico de mi tesis doctoral sobre las estrategias económicas en el uso de las remesas de la migración ecuatoriana en España, y los factores sociales que influyen en estos envíos de dinero tanto en el país de origen como en el de destino ${ }^{3}$.

\footnotetext{
${ }^{3}$ Para la selección de la muestra en la que se basa la información aquí presentada se intentó tener accesos muy diferentes a las personas entrevistadas con el fin de garantizar en la medida de lo posible la pluralidad de ésta. Igualmente, para garantizar la pluralidad y representatividad de la muestra se adoptaron varios criterios generales: 1) primar que las relaciones establecidas entre los contactos no fuesen familiares sino que estuviesen establecidas en otros ámbitos (por ejemplo, relaciones de amistad o trabajo); 2) no entrevistar a más de dos personas proporcionadas por un mismo contacto; y 3) evitar en la medida de lo posible las cadenas de contactos (un contacto lleva a otro y así sucesivamente) para primar los accesos por diferentes vías a las personas entrevistadas.

Igualmente, se tomaron como variables el sexo, la edad, el tiempo de estancia en España, la situación legal, la procedencia de origen (rural o urbana y Sierra o Costa), la ciudad de destino y la existencia de familiares dependientes económicamente, el grado de parentesco en relación al familiar dependiente, así como el lugar de residencia (en Ecuador o en España) de dicho familiar dependiente. Con todo ello, la muestra final se compuso de 22 migragramas en el conjunto de la muestra hay 11 hombres y 11 mujeres entrevistados. De ellos 16 provienen de la Sierra y 6 de la Costa, mientras que 17 provienen de zonas urbanas y 5 de zonas rurales. En cuanto a la edad, 7 tenían menos de 30 años en el momento de ser entrevistados, 12 tenían entre 30 y 44 años y 3 tenían
} 
Para la realización de un trabajo así, la encuesta no parecía el método de investigación más idóneo por su naturaleza: para la operacionalización y análisis posterior, la encuesta como técnica cuantitativa, reduce y estandariza los datos que ha recogido a la vez que aísla a éstos del contexto social en el cual se producen.

Por ello, decidí inclinarme por realizar un protocolo de investigación propio basado en el análisis de redes, aunque con algunas ligeras variaciones para adaptarlo a mi interés sobre los envíos de remesas ${ }^{4}$. Las ventajas de esta técnica de investigación residían en su capacidad para operar con unos datos en los que se realiza una reducción débil de la realidad y, al mismo tiempo, posibilitar una operacionalización fuerte de los datos obtenidos. Esto contrastaba con las posibilidades de técnicas cualitativas como la entrevista o la observación, técnicas que ofrecen una reducción débil de la realidad pero al mismo tiempo unas posibilidades de operacionalización también débiles. Y, a su vez, contrastaba con otras técnicas cuantitativas como la encuesta, un instrumento que ofrece unas posibilidades fuertes de operacionalización de los datos pero análogamente realiza una fuerte reducción de la realidad.

Sin embargo, durante la recogida de datos se hicieron patentes algunas debilidades en torno a este instrumento metodológico relacionadas con el objeto de estudio a analizar. Estas debilidades se relacionaban con el análisis de los cambios que se iban produciendo en las pautas de los envíos de dinero y en las cantidades enviadas, unos cambios que se hacían más evidentes cuando se recogían casos de personas que llevaban un tiempo prolongado residiendo en España y cuya perspectiva de retorno a Ecuador o de permanencia en España había variado considerablemente durante todo este tiempo. En muchos de estos casos la información oral recogida evidenciaba que a lo largo del tiempo se habían producido importantes variaciones en las remesas enviadas, lo que chocaba con las limitaciones que ofrecían las plantillas de la red migratoria para sistematizar los datos obtenidos, puesto que ésta se refería únicamente al momento de realización de la entrevista. Con ello, se hacía necesaria la adopción de una perspectiva procesual en torno a la evolución existente en los envíos de dinero realizados.

A su vez, las redefiniciones continuas que se iban observando en algunas entrevistas sobre la perspectiva de retorno a Ecuador (o de permanencia en España) y la pluralidad de situaciones encontradas entre los migrantes aconsejaba abordar el estudio de las relaciones e interacciones mutuas existentes entre la perspectiva de retorno o de permanencia del migrante y el envío de remesas así como los factores más determinantes que influían sobre estas cuestiones. Igual-

más de 45 años. Igualmente, de todas las personas entrevistadas únicamente 2 declaraban no enviar remesas mientras que 20 sí lo hacían. Dentro de estos, 10 enviaban remesas con mayor o menor periodicidad a hijos o hermanos, 18 lo hacían a padres o abuelos, y 11 enviaban remesas que iban destinadas a algún tipo de inversión.

${ }^{4}$ Dicha propuesta puede verse en Sanz Abad (2009). 
mente, se hacía útil buscar algún instrumento metodológico que pudiese recopilar datos sobre estas cuestiones desde una perspectiva comparada.

El resultado de todo ello fue un protocolo de recogida de datos denominado migragrama (ver Figura 1).

El migragrama es una herramienta metodológica con la que se pretende realizar una vinculación entre el envío y utilización de las remesas, por un lado, y el proyecto migratorio y los acontecimientos que se suceden tanto en el país de origen como de destino, por otro. Esta asociación permite establecer vínculos entre los envíos de remesas, el campo social migratorio en el que éstas se producen y el marco más amplio de las estrategias económicas seguidas por los migrantes. Para ello, se parte de un enfoque procesual que permite captar una visión longitudinal del proceso migratorio, y que presta atención a las incidencias y modificaciones que se pueden producir en el entorno personal y/o familiar.

Dentro de la plantilla del migragrama existen dos dimensiones perfectamente diferenciadas:

- La parte superior de la plantilla está encaminada a recoger información sobre algunos acontecimientos sucedidos a nivel biográfico y en el entorno familiar en España y en Ecuador. El fin de recopilar esta información es analizar las relaciones existentes entre estos sucesos y la importancia que éstos tienen en las posibles redefiniciones que se den del proyecto migratorio. En esta parte existen varias secciones:

1. En primer lugar aparece el espacio dedicado a los acontecimientos biográficos, donde se anotan todos aquellos sucesos acaecidos al migrante desde que éste emprendió el viaje que, no están directamente relacionados ni con su país de origen ni con el de destino, y que dada su relevancia han podido modificar el proyecto migratorio inicial (por ejemplo, el nacimiento de un hijo o una enfermedad de cierta gravedad).

2. En la segunda sección del migragrama se recogen a partir de algunas categorías especificadas, algunos sucesos que han podido acontecer durante la migración tanto en el país de origen como en el de destino. Así, en los acontecimientos sucedidos en España se recopila información sobre la evolución del status jurídico del migrante en España, sobre su situación laboral (trabajos realizados y posibles cambios en los ingresos salariales que se hayan podido dar), y sobre su situación residencial (domicilio en el mismo lugar de trabajo, alquiler de habitación o de vivienda completa o vivienda en propiedad, número de personas con las que vive, etc.), así como sobre otras posibles inversiones realizadas por el migrante en España.

Para complementar estos datos, en los acontecimientos sucedidos en Ecuador se recopila información que aporta indicios sobre los vínculos que el migrante mantiene con su país de origen (viajes a 
Ecuador realizados), así como otros datos referentes a algunos acontecimientos sucedidos mientras él había migrado (enfermedades, fallecimientos de familiares, etc.) y sobre la realización de reagrupaciones familiares que se hayan podido realizar durante este tiempo.

3. Finalmente, en la parte superior del migragrama aparece la categoría del proyecto migratorio, concepto con el que me refiero a la perspectiva temporal definida o indefinida que el migrante tiene de retorno al país de origen o de permanencia en el país de acogida en un momento concreto de la migración (en el momento de la migración, pasados 5 años, etc.). De este modo, el proyecto migratorio debe ser entendido de una manera procesual a la vez que en muchas ocasiones éste se vincula a un fin concreto y se liga a la movilidad social del migrante o de su unidad familiar.

Además, a través de la utilización de flechas se pretende vincular y enfatizar las relaciones entre los diferentes acontecimientos que se suceden y las modificaciones que se producen en el proyecto migratorio.

Con ello, la finalidad de partir de estas categorías es realizar una recogida de datos que aborde las posibles relaciones existentes entre algunos acontecimientos sucedidos tanto en el país de origen como en el de destino y el proyecto migratorio, así como resaltar el carácter procesual de dicho proyecto y las constantes redefiniciones que se pueden dar del mismo en función de diferentes acontecimientos. Así, el proyecto migratorio se presenta como la consecuencia de una serie de elementos que se dan tanto en el país de origen como en el de destino y que influyen en éste actualizándolo, ya sea manteniéndolo, modificándolo o redefiniéndolo.

- El migragrama se ve completado en su parte inferior con los datos recogidos referentes a los envíos de remesas que realiza el informante y la evolución que han ido teniendo éstos a lo largo del tiempo. La información recogida está dividida en una primera sección sobre envíos de dinero (en la que se realizan anotaciones de tipo general sobre los envíos que se realizan al país de origen) y, posteriormente, en cuatro apartados destinados a analizar la finalidad que se da a estos envíos: deudas, envíos a familiares, inversiones y ahorro:

1. En el apartado de deudas, se abordan los envíos de dinero que se han realizado con el fin de pagar deudas adquiridas por el migrante o por otro familiar, preferentemente relacionadas con la movilización de recursos necesaria para efectuar la migración.

2. La sección de envíos a familiares va destinada a cubrir información sobre los envíos que van dirigidos en general hacia algún familiar. En esta sección se distingue entre gastos diarios (aquellos que van destinados básicamente a cubrir gastos de manutención), envíos para 
salud (envíos para medicinas, terapias, etc.), envíos destinados a educación, y otros envíos para cubrir gastos eventuales (aquellos que surgen puntualmente como entierros, fiestas u otras celebraciones) ${ }^{5}$.

3. Una tercera sección analiza los envíos de dinero que van destinados a cubrir aquellas inversiones que en muchos trabajos son conceptualizadas como productivas. En este apartado he seguido un criterio muy similar al expresado por Lozano Ascencio (2000), quien identifica tres formas de inversión productiva. El primer apartado (vivienda) se corresponde con lo que este autor identifica como inversión en bienes duraderos como la compra de terrenos, la compra o la remodelación de una casa. Mientras tanto, en el apartado de otras inversiones (tanto propias como de otros) se englobarían las otras dos formas que este autor señala: inversión en bienes de capital, como la compra de vehículos, maquinaria y herramientas, y la formación de pequeños negocios y empresas.

4. Finalmente, un último apartado está destinado a aquellos envíos de dinero que tienen como finalidad el ahorro en Ecuador.

Con ello, lo más singular del migragrama es la vinculación que se realiza entre el envío y utilización de las remesas por un lado, y el proyecto migratorio y los acontecimientos que se suceden tanto en el país de origen como de destino por otro, lo que permite establecer desde un enfoque procesual vínculos entre los envíos de remesas, el campo social migratorio en el que éstas se producen y el marco más amplio de las estrategias económicas seguidas por los migrantes cuestiones éstas que normalmente son analizadas por separado.

${ }^{5}$ En muchos de los trabajos que se realizan sobre los envíos de remesas existe cierta controversia sobre cómo conceptualizar los gastos destinados a educación y a salud, puesto que, en ocasiones, estas partidas se consideran como consumo de bienes básicos, mientras que otras veces son consideradas como inversión en capital humano (al considerarse que estas partidas sirven para valorizar la fuerza de trabajo). Finalmente, en otras circunstancias algunos autores diferencian entre la educación y la salud conceptualizando a la primera como inversión en capital humano y a la segunda como gasto.

Ante la controversia existente, y aun inclinándome personalmente por la última opción, dada la importancia que revisten las partidas destinadas a educación y salud respecto a aquellos destinados a cubrir gastos diarios u otros gastos de carácter puntual (como entierros, celebraciones, etc.), he optado por incluir estas partidas en el apartado de envíos a familiares, aunque desglosando estas partidas de los envíos destinados a cubrir gastos de manutención u otros gastos más esporádicos. 


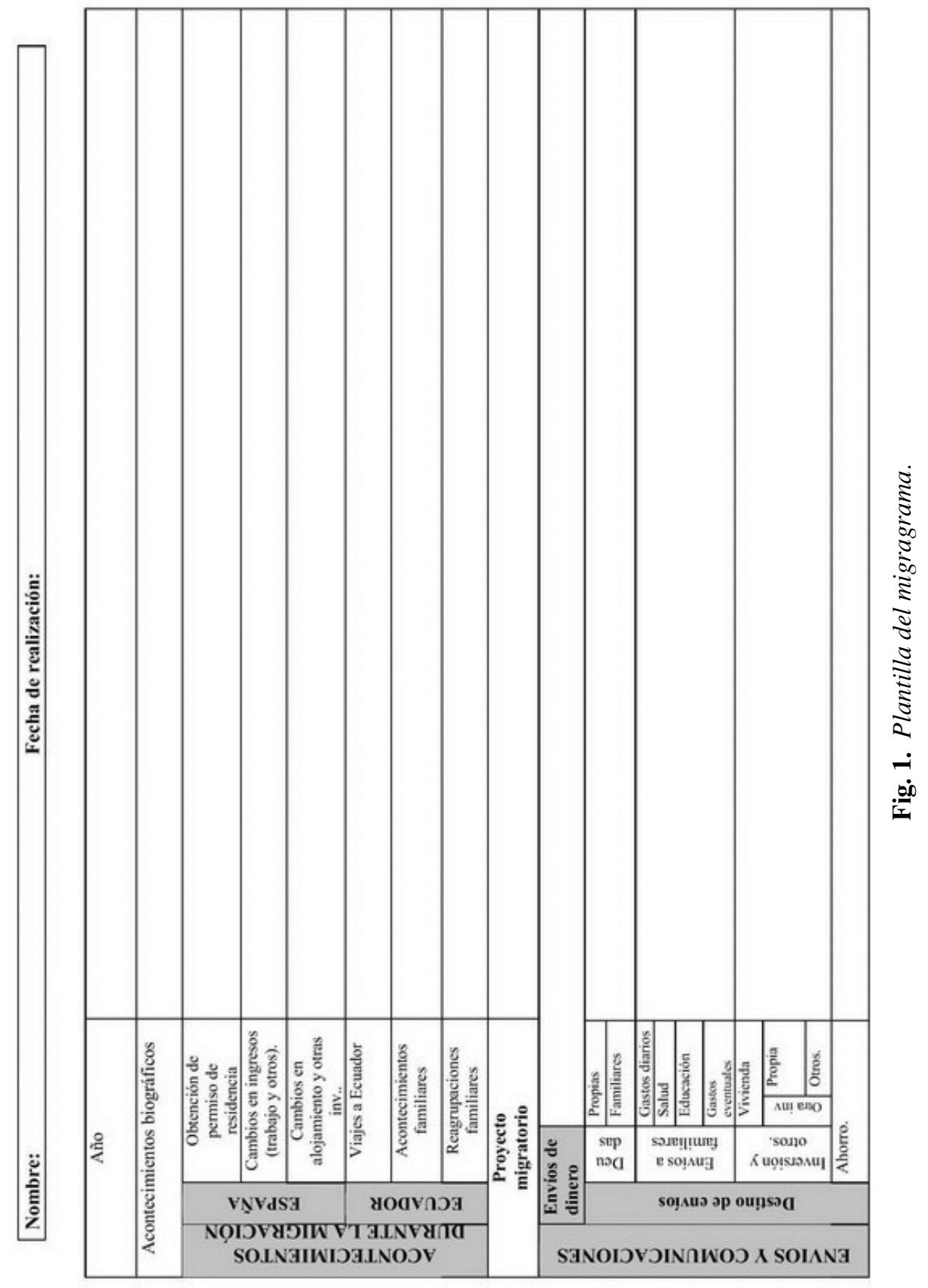

EMPIRIA. Revista de Metodología de Ciencias Sociales. N. ${ }^{\circ}$ 19, enero-junio, 2010, pp. 207-232. ISSN: 1139-5737 


\section{Ventajas de la propuesta del migragrama}

Más allá de la utilidad del migragrama como representación gráfica que permite captar de manera sintética diferentes aspectos del proceso migratorio, las características de esta técnica son útiles por varias razones para abordar el estudio de las remesas.

- En primer lugar, el migragrama, recoge una visión longitudinal del proceso migratorio, lo que permite estudiar la migración desde una perspectiva procesual alejada de las perspectivas unidireccionales centradas en la asimilación como única estrategia de los migrantes. Como señala Suárez (2007b), la perspectiva transnacional enfatiza una visión de los migrantes como sujetos móviles con lógicas de pertenencia incompletas, algo que concuerda muy bien con la metodología del migragrama con su énfasis en el carácter procesual de la migración y la redefinición del proyecto migratorio como consecuencia de una sucesión de acontecimientos que influyen en él.

- En segundo lugar, el migragrama permite analizar las remesas en el marco más amplio de las estrategias económicas seguidas por los migrantes. Así, la vinculación entre los acontecimientos sucedidos durante el periodo migratorio y las variaciones que se van produciendo en los envíos de remesas permite establecer relaciones sobre los factores que más influyen en los usos y cantidades de dinero enviado.

De este modo, el migragrama se convierte en una herramienta de análisis útil para captar aspectos como la movilidad social durante el proceso migratorio, las diferentes estrategias económicas seguidas por los migrantes y sus familias (el migragrama aporta indicios sobre la primacía del ahorro o el gasto en estas estrategias económicas), o la relación que existe entre estas estrategias económicas y el marco espacial en el que se piensan llevar a cabo las estrategias de movilidad social (Ecuador o España).

- En tercer lugar, el migragrama permite establecer relaciones entre la importancia que los migrantes conceden a enviar dinero a su país, las formas de inserción y las condiciones de vida que tienen en España. Esta cuestión es importante si se tiene en cuenta que el estudio de los envíos de dinero y el estudio de las formas de vida de los migrantes, generalmente son abordados de forma separada y no de forma conjunta, En contraste, la información obtenida a partir del migragrama ayuda a entender las prioridades que tienen los migrantes. En este sentido es importante reseñar que, a partir de los datos recogidos en diferentes trabajos basados en esta metodología (Sanz Abad, 2008) parece existir una fuerte relación entre las estrategias económicas, laborales, residenciales (tanto en el tipo de vivienda que se habita como en el tipo de localidad) adoptadas por los migrantes (e incluso en algunos casos, también, entre las estrategias desarrolladas a nivel formativo y de aprendizaje del idioma) y la perspectiva de permanencia o de retorno del migrante.

- Por último, el migragrama permite ofrecer una perspectiva comparada de diferentes casos derivada del carácter estandarizado de recogida de datos cua- 




EMPIRIA. Revista de Metodología de Ciencias Sociales. N. ํ 19, enero-junio, 2010, pp. 207-232. ISSN: 1139-5737 


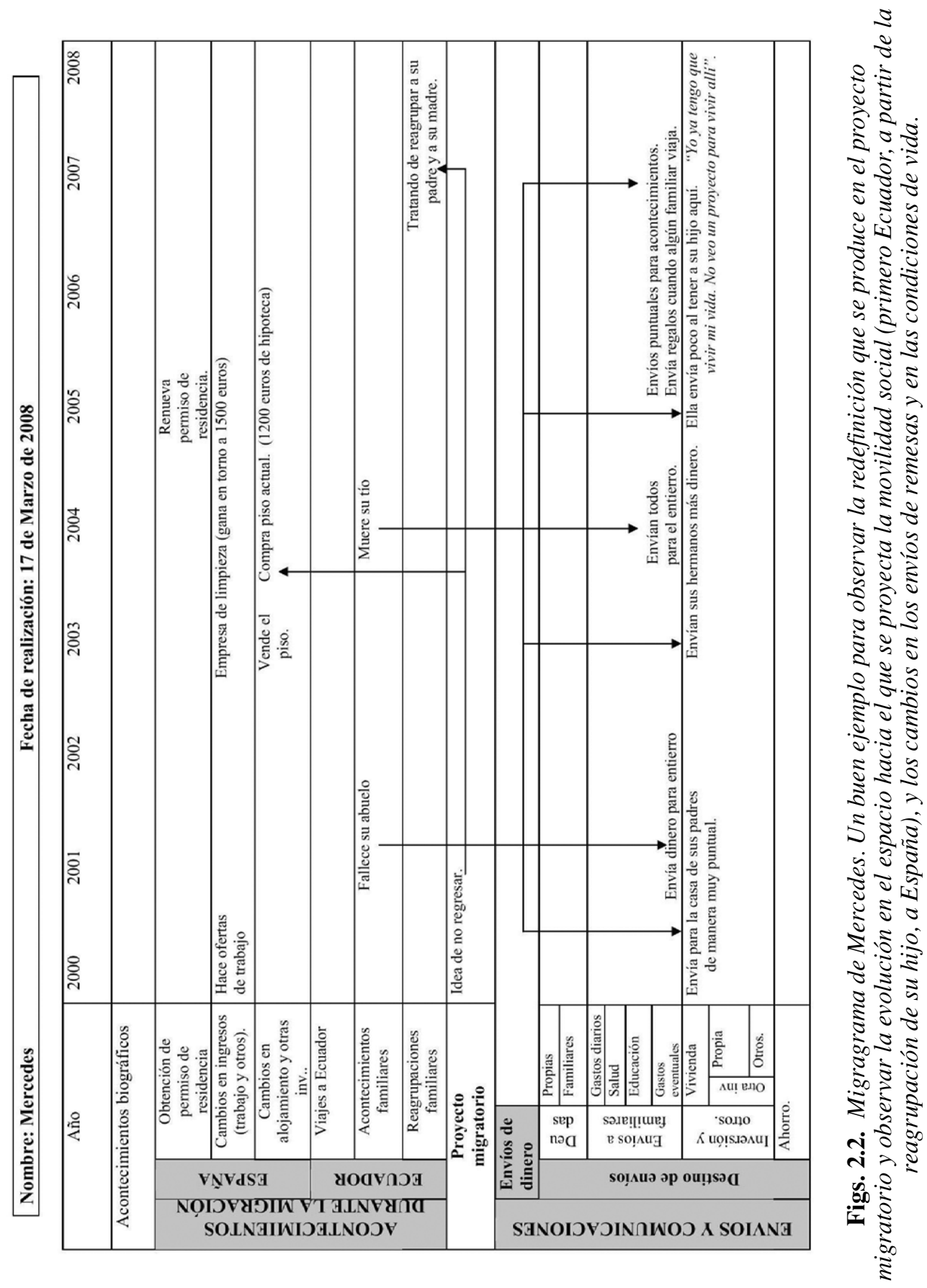

EMPIRIA. Revista de Metodología de Ciencias Sociales. N. ํ 19, enero-junio, 2010, pp. 207-232. ISSN: 1139-5737 




EMPIRIA. Revista de Metodología de Ciencias Sociales. N. ${ }^{\circ}$ 19, enero-junio, 2010, pp. 207-232. ISSN: 1139-5737 
litativos que tiene este instrumento, ya sea entre personas de una misma nacionalidad o entre personas de diferentes colectivos ${ }^{6}$.

Esta perspectiva comparada permite mostrar la heterogeneidad de situaciones que existen en torno a la migración, a la vez que sirve para reflejar la variedad de formas diferentes en las que los migrantes pueden «estar»o «pertenecer» en el territorio (Levitt y Glick Schiller, 2004). Del mismo modo, este marco comparativo sirve para reflejar la pluralidad de estrategias económicas y de situaciones referentes a la perspectiva de retorno o permanencia que existen.

Mostrar esta heterogeneidad y aportar elementos que complejicen y favorezcan una mejor comprensión del fenómeno migratorio es importante si se tiene en cuenta que frecuentemente se enfatiza el carácter exclusivamente económico de la migración, y la tendencia hacia la asimilación por parte de los migrantes como única forma de inserción en el país de destino.

\section{Otros hallazgos y ventajas del migragrama descubiertos a partir del trabajo de campo}

Además de las cuestiones a las que el migragrama como técnica de investigación pretendía dar respuesta en los momentos previos a su utilización, durante la recogida de datos aparecieron algunas ventajas adicionales que esta técnica de investigación tiene en relación con otras.

- Una primera cuestión que se reveló útil durante la realización del trabajo de campo se deriva de las posibilidades que ofrece el migragrama para articular y relacionar los datos recogidos a nivel micro con algunos acontecimientos que suceden a nivel macro (procesos extraordinarios de regularización, crisis económicas, etc.). Esta perspectiva permite conocer de primera mano cómo algunos acontecimientos que se producen a nivel macro afectan a las trayectorias migratorias y a las estrategias seguidas por los migrantes, así como permite conocer más sobre las posibles respuestas que los migrantes dan a estas eventualidades. Así, la posibilidad de relacionar estos acontecimientos macro con informaciones sobre las trayectorias y cambios laborales, residenciales, y/o en la realización de inversiones o los envíos de dinero realizados, aporta una información suplementaria que permite conocer mejor las lógicas de actuación de los migrantes.

- Una segunda ventaja se deriva de las posibilidades que existen al combinar el migragrama con otras técnicas de investigación como la entrevista semiestructurada, dado que el conjunto de datos que se sistematiza a través del migragrama, supone una excelente herramienta de elicitación que fomenta la

${ }^{6}$ En Sanz Abad (2009) esta técnica es utilizada en el análisis de la migración ecuatoriana en España en momentos de la migración muy diferentes. Por su parte, en Sanz Abad (2008) se realiza un análisis de los colectivos ecuatoriano, marroquí y rumano a partir del análisis de diferentes casos recogidos en Castilla-La Mancha.

EMPIRIA. Revista de Metodología de Ciencias Sociales. N. ํ 19, enero-junio, 2010, pp. 207-232. ISSN: $1139-5737$ 
producción de discurso por parte del entrevistado a partir de la exploración conjunta de la reconstrucción de la experiencia migratoria sobre la base del diálogo y los recuerdos.

- Finalmente, y en relación con el estudio de los envíos de dinero, una tercera cuestión relacionada con la utilización del migragrama y que es de gran interés es la visibilización de determinados envíos de dinero sobre los que sospecho que otros instrumentos metodológicos, como las encuestas, no muestran o minusvaloran. Para comprender esta cuestión, hay que analizar qué se entiende por remesa desde la contabilidad por un lado, y qué entienden por remesa generalmente los migrantes, una cuestión que como se verá es bastante compleja.

Dentro de la contabilidad, los registros de las balanzas de pagos pueden presentar tres conceptos relacionados con las remesas: remesas de trabajadores (worker remittances), compensación de empleados (compensation of employees) y a un nivel mayor de desagregación, las transferencias de migrantes (migrant transfers $)^{7}$ (Alonso, 2004). De estos conceptos, el concepto que generalmente se ha equiparado en la mayoría de los casos al concepto de remesas, es el de remesa de trabajadores (workers' remittances), entendiendo por ello las transferencias corrientes de los extranjeros residentes, que han permanecido o intentan permanecer por un período de por lo menos un año ${ }^{8}$.

Sin embargo, el nuevo Manual del Fondo Monetario Internacional en su sexta y última edición de diciembre de $2008^{9}$ matiza y complejiza esta cuestión. El FMI señala que no hay un ítem específico en la estructura de la balanza de pagos que permita mostrar el conjunto de las remesas, y considera que éste debe ser entendido como «la suma de las 'remesas personales' y los subsidios sociales, incluyendo los subsidios sociales, los beneficios pagables bajo fondos de la Seguridad Social y Fondos de Pensiones, y pudiendo ser éstos en efectivo o en especie» ${ }^{10}$ (FMI, 2008: 405).

El Manual continua explicando cómo las remesas personales incluyen «los ingresos de los trabajadores por cortos periodos de tiempo (incluidos en compensation of employees), los ingresos de los trabajadores residentes en el extranjero ("personal transfers), esto es, las transferencias corrientes, en efectivo o en especie realizadas por los hogares de residentes a los hogares de no residentes, sin tener en cuenta las fuentes de los ingresos del emisor, ni la relación entre los hogares, ni el motivo de la remesa $»^{11}$, y las transferencias de capital

7 A las dificultades conceptuales hay que sumar además la opacidad que buena parte de los canales de transferencia presentan al control estadístico y cuyas estimaciones conservadoras indican que llegarían a suponer al menos un tercio de las remesas registradas (Alonso, 2004).

8 Así hace el Banco de España (remesas.org, 2008).

9 Dicho Manual sirve de referencia a nivel mundial para fijar conceptos relacionados con cada registro de la balanza de pagos.

10 Traducción personal.

11 El Manual del FMI aclara que la partida de «transferencias personales» es estándar en las balanzas de pagos, siendo las otras partidas adicionales. 
(los flujos de bienes y los cambios en rubros financieros que surgen por la migración de individuos de una economía a otra). (FMI, 2008:405-406), A esta partida habría que añadir los subsidios sociales del extranjero que, como se señala en el Manual, «son una partida adicional en la estructura de la balanza de pagos dentro de los ingresos secundarios» (FMI, 2008: 406).

Por todo ello, la suma total de remesas según el Manual del FMI no se corresponde con una partida concreta de la balanza de pagos, sino con un conjunto de partidas como se muestra en la tabla 1.

Tabla 1. Conjunto de remesas según el manual del FMI (2008)

\begin{tabular}{|l|l|l|}
\hline \multicolumn{2}{|c|}{ Remesas totales Remesas personales + subsidios sociales $(\mathbf{a}+\mathbf{b}+\mathbf{c}+\mathbf{d})$} \\
\hline \multicolumn{2}{|c|}{ Remesas personales $=\mathbf{a}+\mathbf{b}+\mathbf{c}$} & \multirow{2}{*}{$\begin{array}{c}\text { Subsidios } \\
\text { sociales }\end{array}$} \\
$\begin{array}{l}\text { a) Transferencias per- } \\
\text { sonales }\end{array}$ & $\begin{array}{l}\text { b) Compensaciones de } \\
\text { empleados menos im- }- \\
\text { puestos, aportaciones } \\
\text { sociales, transporte y } \\
\text { viajes }\end{array}$ & $\begin{array}{l}\text { capital } \\
\text { viajerencias de }\end{array}$ \\
\hline
\end{tabular}

Fuente: Manual del FMI (2008).

La cuestión se complica más aún al contrastar el uso que en el ámbito contable se hace del concepto de remesas con el uso coloquial de este concepto. Como señala remesas.org (2008), en el lenguaje coloquial se entiende por remesas el dinero que los emigrantes envían a su país de origen, siempre que sea a cambio de nada tangible.

Sin embargo, en relación con esta definición no está nada claro lo que se entiende por «algo tangible» dado que las casuísticas que pueden aparecer son muy amplias. Matizando aun más la afirmación de remesas.org, y a partir de la experiencia recogida en el trabajo de campo realizado con migrantes ecuatorianos, considero que la mayor parte de las veces, cuando los migrantes son preguntados por «el dinero que envían» ${ }^{12}$, éstos tienden a asociar sus envíos de remesas eminentemente con el dinero que tiene como destinatario último a una persona concreta (diferente al propio emisor ${ }^{13}$ ) y sobre todo si esos envíos son hechos de manera periódica y regular, o si esos envíos de dinero son realizados de manera esporádica y van destinados a cubrir algún gasto extraordinario asociado a algún acontecimiento puntual (enfermedad, alguna celebración, etc.). Si-

12 El entrecomillado es mío. Con él pretendo enfatizar que los migrantes normalmente hablan más del «dinero que envían», que de las remesas (un término que sería más bien una categoría «etic»).

${ }^{13}$ Como se muestra más abajo, en ocasiones la compra de una vivienda en Ecuador, cuyo destinatario final es el propio migrante, no es considerada como remesa. 
guiendo esta argumentación, a partir de los datos recogidos se observó cómo en muchas ocasiones no se consideraban como remesas entre otros, algunos envíos ligados a cubrir el pago de deudas derivados de la migración, o las inversiones y gastos que se realizan aprovechando algún viaje a Ecuador.

Con ello, es desde esta interpretación émica de lo que el migrante entiende por remesa, desde donde se explicarían algunos comentarios y situaciones concretas encontradas en el trabajo de campo que expongo:

- El caso de un informante quien durante una entrevista comentaba que nunca había enviado dinero «a su casa» ${ }^{14}$, y al ser preguntado si había hecho algún tipo de inversión con el dinero ahorrado durante su estancia en España indicaba que había comprado un taxi con el que su hermano trabajaba en Quito.

- El caso de una persona que declaraba enviar unos 300 euros al mes a su familia, pero que al ser preguntada sobre su último viaje a Ecuador, afirmaba que sus empleadores la habían adelantado un año de sueldo ${ }^{15}$ y que con ese dinero había pagado buena parte de una vivienda comprada aprovechando un viaje a Ecuador ${ }^{16}$.

- El contraste entre la escasez de datos que aparecen en los migragramas sobre dinero enviado a sufragar deudas ligadas a la provisión de recursos para migrar tanto del migrante como de otras personas, y la abundancia de datos recogidos a partir de la utilización del análisis de redes migratorias, que indican la existencia de múltiples envíos de dinero realizados con el fin de posibilitar que nuevas personas migren.

- La no consideración como remesa por parte de los migrantes de aquellas que he denominado «remesas indirectas». Con este término me refiero a la utilización regular que alguna persona en Ecuador puede realizar de los rendimientos obtenidos por medio de una inversión (como por ejemplo el arriendo de una vivienda o un local) que ha sido financiada con los envíos de dinero realizados durante la migración, una práctica que aparece especialmente entre los migrantes que llevan más tiempo residiendo en España. Así sucedía, por ejemplo, con el dinero procedente del arriendo de una casa en Quito que recibía y disfrutaba la madre de una migrante, cuya propietaria en realidad era su hija que residía en Madrid.

${ }^{14}$ Refiriéndose a sus familiares.

15 Hay que tener en cuenta que esta mujer trabajaba en el servicio doméstico como interna, situación que favorece la existencia de unas relaciones entre empleador y empleada más flexibles a lo pactado en el contrato de trabajo.

${ }^{16}$ En el caso concreto de la migración ecuatoriana, frecuentemente se aprovecha la realización de un viaje al país de origen para portar el dinero necesario para la realización de alguna inversión, $\mathrm{y}$ aprovechar la estancia para realizar algunas tomas de decisiones que afectan a estas inversiones (y por tanto, estos envíos de dinero serían remesas informales que rara vez son estimadas por la mayor parte de trabajos realizados).

EMPIRIA. Revista de Metodología de Ciencias Sociales. N. . 19, enero-junio, 2010, pp. 207-232. ISSN: $1139-5737$ 
- La no consideración como remesa por parte de los migrantes de las compras de viviendas en Ecuador realizadas desde España. Esta ausencia de consideración como remesa es especialmente frecuente cuando el destinatario principal de la compra es el propio migrante, y si esa vivienda no es de autoconstrucción, sino que es realizada por una empresa inmobiliaria.

Con ello, en la tabla 2 realizo una aproximación a las diferentes acepciones que maneja la contabilidad, los migrantes, así como algunas consideraciones personales sobre este concepto de las que partía en el trabajo de campo realizado.

Pero más allá de las diferencias encontradas, me interesa subrayar sobre todo, los efectos que se pueden derivar de esta diferente conceptualización de la noción de remesa.

La técnica de investigación a la que más se recurre para recoger datos referentes sobre las cuantías enviadas a través de las remesas o los usos y finalidades que los migrantes y sus familias dan a éstas es la encuesta. Pues bien, sin negar las evidentes ventajas que esta técnica presenta (posibilidades de operacionalización fuerte así como de generalización de los datos obtenidos), a mi juicio existe un riesgo implícito en el uso de esta técnica relacionado con la conceptualización de lo que el migrante entiende por remesa.

Cuando se pregunta al migrante o al familiar sobre las remesas enviadas o recibidas, normalmente éste privilegia en sus respuestas aquellos envíos que se realizan de una manera regular en el tiempo. Esta cuestión puede ser reforzada más todavía si la pregunta realizada enfatiza el promedio de dinero enviado (como por ejemplo si se pregunta En promedio, ¿cuánto dinero le envía usted a su familia cada vez?»).

Se da la circunstancia de que generalmente los envíos periódicos suelen ir destinados en la mayor parte de los casos a cubrir gastos diarios y otros gastos fijos más o menos periódicos como los gastos en salud y en educación (especialmente, si en la formulación de la pregunta en la encuesta se desglosa el monto destinado a salud y educación del monto destinado a gastos diarios, ya que muchas veces estos apartados no están desagregados).

Con ello, al utilizar únicamente la encuesta como método de recogida de datos se corre el riesgo de no tener en cuenta o de minusvalorar aquellos envíos de dinero que no se realizan de manera regular sino eventualmente, y que en algunos casos pueden adquirir gran importancia. Los datos recogidos en el trabajo de campo indicaban que este tipo de remesas más ocasionales estaban muchas veces ligadas a inversiones (compra o construcción de vivienda, inversión en bienes de equipo, etc.), a atender situaciones imprevistas a través de las cuales el migrante expresa su solidaridad (enfermedades, costear los gastos de un entierro, etc.), a costear los gastos para que otra persona pueda migrar (pago del billete de avión, «bolsa de viaje», etc.), o a la realización de celebraciones concretas que son significativas para el migrante (bodas, bautizos, fiestas, etc.). 
Tabla 2. Una aproximación a la conceptualización del concepto de remesa desde diferentes ámbitos

\begin{tabular}{|c|c|c|c|c|}
\hline & Variable & Contabilidad $^{17}$ & Migrantes ecuatorianos $^{18}$ & Visión del autor \\
\hline \multirow{2}{*}{$\begin{array}{l}\text { Según la } \\
\text { periodicidad } \\
\text { de envío }\end{array}$} & Periódica & $\begin{array}{l}\text { Se contabiliza } \\
\text { como remesas* }\end{array}$ & Se considera remesa & $\begin{array}{l}\text { Se considera } \\
\text { remesa }\end{array}$ \\
\hline & Esporádica & $\begin{array}{l}\text { Se contabiliza } \\
\text { como remesas* }\end{array}$ & $\begin{array}{l}\text { Varía en función de la } \\
\text { situación** }\end{array}$ & $\begin{array}{l}\text { Se considera } \\
\text { remesa }\end{array}$ \\
\hline \multirow[b]{2}{*}{$\begin{array}{l}\text { Según el } \\
\text { canal de } \\
\text { envío }\end{array}$} & $\begin{array}{c}\text { Regular (transferencias, } \\
\text { etc.). }\end{array}$ & $\begin{array}{l}\text { Se considera } \\
\text { remesas }\end{array}$ & En función de la finalidad. & $\begin{array}{l}\text { Se considera } \\
\text { remesa }\end{array}$ \\
\hline & $\begin{array}{c}\text { Irregular } \\
\text { (aprovechamiento de } \\
\text { algún viaje para } \\
\text { trasladar dinero o envíos } \\
\text { de mensajería) }\end{array}$ & $\begin{array}{c}\text { No se } \\
\text { contabiliza }\end{array}$ & $\begin{array}{l}\text { Depende del canal de envío } \\
\text { (Si el dinero se lleva } \\
\text { aprovechando un viaje del } \\
\text { mismo migrante, no suele } \\
\text { ser considerado remesa)** }\end{array}$ & $\begin{array}{l}\text { Se considera } \\
\text { remesa }\end{array}$ \\
\hline \multirow{6}{*}{$\begin{array}{l}\text { Según la } \\
\text { finalidad }\end{array}$} & $\begin{array}{l}\text { Envíos para gastos } \\
\text { diarios }\end{array}$ & $\begin{array}{l}\text { Se contabiliza } \\
\text { como remesa* }\end{array}$ & $\begin{array}{c}\text { Se considera remesa } \\
\text { normalmente }\end{array}$ & $\begin{array}{l}\text { Se considera } \\
\text { remesa }\end{array}$ \\
\hline & $\begin{array}{l}\text { Pago de deudas y dinero } \\
\text { ligado a la provisión de } \\
\text { recursos para migrar }\end{array}$ & $\begin{array}{l}\text { Se contabiliza } \\
\text { como remesa* }\end{array}$ & $\begin{array}{c}\text { No se asocia normalmente a } \\
\text { una remesa }\end{array}$ & $\begin{array}{l}\text { Se considera } \\
\text { remesa }\end{array}$ \\
\hline & $\begin{array}{c}\text { Inversiones en vivienda } \\
\text { u otros (inversión en } \\
\text { bienes de capital). }\end{array}$ & $\begin{array}{l}\text { Puede no ser } \\
\text { contabilizado } \\
\text { como remesa* }^{*}\end{array}$ & $\begin{array}{l}\text { Según casos (si la vivienda } \\
\text { es para el mismo migrante y } \\
\text { sobre todo si no es de } \\
\text { autoconstrucción muchas } \\
\text { veces no es conceptualizado } \\
\text { como remesa)** }\end{array}$ & $\begin{array}{l}\text { Se considera } \\
\text { remesa }\end{array}$ \\
\hline & $\begin{array}{l}\text { Dinero enviado ligado a } \\
\text { un negocio en destino }\end{array}$ & $\begin{array}{l}\text { Se contabiliza } \\
\text { como remesa* }\end{array}$ & $\begin{array}{l}\text { No es considerado como } \\
\text { remesa }\end{array}$ & $\begin{array}{l}\text { En función de la } \\
\text { definición de } \\
\text { remesa de la que } \\
\text { se parta }\end{array}$ \\
\hline & $\begin{array}{l}\text { Beneficios extraídos del } \\
\text { arriendo de un bien } \\
\text { inmueble }\end{array}$ & $\begin{array}{c}\text { No se } \\
\text { contabiliza } \\
\text { como remesa }\end{array}$ & $\begin{array}{l}\text { No se considera como } \\
\text { remesa }\end{array}$ & $\begin{array}{l}\text { Remesa } \\
\text { (ejemplo de } \\
\text { remesa } \\
\text { indirecta) }\end{array}$ \\
\hline & Subsidios sociales & $\begin{array}{l}\text { Se contabilizan } \\
\text { en remesas } \\
\text { totales, pero no } \\
\text { en remesas } \\
\text { personales. }\end{array}$ & No son tenidos en cuenta. & $\begin{array}{l}\text { No es abordado } \\
\text { en el estudio. }\end{array}$ \\
\hline
\end{tabular}

Fuente: Elaboración propia.

${ }_{17}$ Parto de la conceptualización que la 6 . edición del Manual del FMI realiza de las remesas (FMI, 2008).

${ }^{18}$ Esta visión general referida a los migrantes ecuatorianos lógicamente puede variar. Los datos presentados en esta tabla están basados en situaciones concretas encontradas en el trabajo de campo (algunas de estas situaciones han sido presentadas en el texto), por lo que se trata sobre todo de una aproximación orientativa a esta cuestión. formal).

* En función del canal de envío (se consideran remesas siempre y cuando vaya por un canal

** El migrante asocia las remesas sobre todo al envío que realiza de manera periódica y ligado al envío de familiares. En función de cómo sea preguntado sobre los envíos de dinero, el migrante

EMPIRIA. Revista de Metodología de Ciencias Sociales. N. ${ }^{\circ}$ 19, enero-junio, 2010, pp. 207-232. 
Por ello, a partir de la diferente conceptualización de lo que se entiende por remesa, del instrumental en la recogida de datos que se utiliza para analizar el uso y finalidad que se da a estos envíos de dinero, y teniendo en cuenta las diferentes pautas de envío que suelen tener las remesas en función de su finalidad, sostengo que, en líneas generales y al menos en lo estudiado en el caso ecuatoriano $^{19}$, los trabajos realizados sobre el envío de remesas tienden a sobrevalorar los envíos destinados a cubrir gastos diarios (especialmente si los gastos de salud y educación no son desglosados de éstos). A su vez estos trabajos tienden a infravalorar e incluso a invisibilizar las remesas que frecuentemente se asocian a envíos esporádicos, y que a su vez suelen estar relacionadas con la realización de inversiones o con la provisión de recursos necesaria para que otra persona migre.

Finalmente y cambiando de asunto, realizaré un último apunte referido a las posibles formas de sistematización que se puede realizar de los datos obtenidos a través del migragrama.

Hasta ahora, en los trabajos realizados con esta herramienta metodológica el análisis que se ha realizado de los datos obtenidos no ha diferido del tratamiento que se hace generalmente de los datos que se consiguen a través de otras técnicas cualitativas como la entrevista o la observación participante dado que el migragrama, al igual que estas técnicas cualitativas, se caracteriza por realizar una reducción y operacionalización débil de la realidad.

En este sentido, en los análisis de los datos obtenidos con esta herramienta que se han hecho en diferentes trabajos (Sanz Abad, 2008; 2009) se ha optado por agrupar estos datos en función de las categorías que se querían analizar, ya sea atendiendo a estas categorías por separado (proyecto migratorio, destino de las remesas, ingresos económicos, etc.) o, como el migragrama posibilita y favorece, estableciendo relaciones entre estas categorías (datos que relacionan de un modo procesual los cambios en el proyecto migratorio con los envío de remesas, etc.). A esta cuestión hay que sumar, también, la utilidad que ha tenido el migragrama como forma de representar e ilustrar a partir de algún caso concreto aquellas afirmaciones que se estaban sosteniendo ${ }^{20}$.

Queda sin embargo por explorar, la sistematización y plasmación que a través de la teoría de grafos se podría hacer de los datos obtenido con los migragramas utilizando para ello las posibilidades que ofrecen los diferentes programas informáticos que se usan frecuentemente en el análisis de las redes sociales. Una cuestión, esta última, que queda abierta para ser abordada en el futuro.

tenderá a conceptualizar estos envíos como remesa con mayor o menor probabilidad. También influye en esta conceptualización el canal utilizado para el envío (no se asocia a remesa aquel dinero que se lleva aprovechando un viaje al país de origen).

${ }_{19}$ En los capítulos 3 y 8 de Sanz Abad (2009) se desarrolla con mayor profundidad esta idea.

${ }^{20}$ En Sanz Abad (2008 y 2009) hay diversos ejemplos en este sentido. 


\section{CONCLUSIONES}

A partir de la emergencia de la perspectiva transnacional, la literatura sobre migraciones ha enfatizado las relaciones que a través de la migración se establecen entre sociedades de origen y destino, así como los procesos y multiplicidad de vínculos existentes entre ambos espacios sociales, siendo las remesas el principal vínculo material existente dentro de este espacio.

Al mismo tiempo, la necesidad de fijarse en varios espacios geográficos y reconocer las interconexiones e interdependencias que se producen en una multiplicidad de lugares como propone la perspectiva transnacional, supone todo un desafío desde el punto de vista metodológico ante el que se hace necesario acudir a nuevas técnicas de investigación y/o nuevas propuestas metodológicas que aporten enfoques y perspectivas novedosas ante determinados objetos de estudio.

En este sentido y como sucede en el caso concreto del estudio de las remesas, el migragrama aquí presentado trata de ser una aportación metodológica que va en esta dirección con su vinculación desde una perspectiva procesual de los envíos de remesas, el campo social migratorio en el que éstas se producen y el marco más amplio de las estrategias económicas seguidas por los migrantes.

\section{BIBLIOGRAFÍA}

Alonso, J. A. (2004): «Emigración y desarrollo: Implicaciones económicas», en Alonso, J. A. Emigración, pobreza y desarrollo. Madrid, Los Libros de la Catarata, pp. 15-70. ApPADURAI, (1996): Modernity at Large: Cultural Dimensions of Global Change. An Anthropological Approach. Londres-Minneapolis, University of Minnesota Press. Trad. española (2001) La modernidad desbordada. Montevideo-Buenos Aires, México, Ediciones Trilce-FCE.

ARANGO, J. (2003): «La explicación teórica de las migraciones: luz y sombra». Migración y Desarrollo, n. ${ }^{\circ} 1$, Octubre de 2003. Disponible en www.migracionydesarrollo.org

FONDO MONETARIO INTERNACIONAL (2008): Balance of payments and international investment Position manual. Sixth Edition (BPM6). Pre-publication Draft, December 2008. Statistics Department, International Monetary Fund. Disponible en: http://www.imf.org/external/pubs/ft/bop/2007/pdf/BPM6.pdf

GARAY, L. J. y RodRIGUEZ, A. (2005): La Migración Internacional: Una Síntesis de Aproximaciones Teóricas Alternativas. La Emigración Internacional en Colombia: Una Visión Panorámica a partir de la Recepción de Remesas. Bogotá, Ministerio de Relaciones Exteriores de Colombia-Organización Internacional para las Migraciones (OIM).

GARCía, M. y PAIEWONSKy, D. (2006): «Género, remesas y desarrollo: El caso de la migración femenina de Vicente Noble, República Dominicana». Santo Domingo, UN - INSTRAW, Instituto Internacional de Investigaciones y Capacitación de las Naciones Unidas para la promoción de la mujer. Disponible en: http://www.un-instraw.org/es/publicaciones/gender-remittances-and-development/index.php. 
HerRerA, G. (2004): «Elementos para una comprensión de las familias transnacionales», en HIDALGO, F. (ed.) Migraciones. Un juego con cartas marcadas. Quito, Ed. Abya-Yala, pp. 215-233.

LEVITT, P (1996): «Social Remittances: A Conceptural Tool for Understanding Migration and Development». Working Paper, Series Number 96-04. Disponible en www.hsph.harvard.edu/hcpds/wpweb/96_04.pdf

- (2001): The Transnational Villagers. Berkeley, University of California Press.

- y Glick Schiller, N. (2004): «Perspectivas internacionales sobre migración: conceptualizar la simultaneidad». Migración y Desarrrollo, Segundo Semestre, 2004 pp. 60-91. Disponible en: www.migracionydesarrollo.org.

LOZANo Ascencio, F. (2000): «Experiencias internacionales en el envío y uso de remesas». Red Internacional de Migración y Desarrollo. Disponible en www.migracionydesarrollo.org

Marcus, G. E. (1995): «Ethnography in/of the World System: The Emergente of Multisited Ethnography». Annual Anthropological Review, n. ${ }^{\circ}$ 24, pp. 96-117. Traducido en (2001) Etnografía en/del sistema mundo. La emergencia de una etnografía multisituada. Alteridades, n. ${ }^{\circ} 11$, pp.111-127.

Martínez PizArro, J. (2005): «La experiencia de la CEPAL en los estudios sobre remesas: lecciones y evidencias». Seminario internacional Problemas y desafíos de la migración y el desarrollo en América, Abril de 2005, Cuernavaca, Morelos. Disponible en www.migracionydesarrollo.org

MOCTEZUMA LONGORIA, M. (2004): «La cultura y el simbolismo de la migración y las remesas. Reflexiones a partir de la experiencia de Zacatecas». Red Internacional de Migración y Desarrollo. Disponible en www.migracionydesarrollo.org.

Ponce, J., OliviÉ, I. y Onofa, M. (2008): Remittances for Development? A Case Study of the Impact of Remittances on Human Development in Ecuador. (Inédito).

REMESAS.ORG (2008a): «¿Qué son las remesas de los inmigrantes?». Disponible en http://www.remesas.org/quesonlasremesas.html

SANZ ABAD, J. (2008): Estudio comparativo del envío de remesas de los principales colectivos extranjeros residentes en Castilla-La Mancha. Estudio realizado para el Centro Asociado de la Universidad Nacional de Educación a Distancia de Guadalajara, y financiado por la Consejería de Bienestar Social de la Junta de Comunidades de Castilla-La Mancha (Inédito).

- (2009): Entre «cumplir» y «hacer cosas». Estrategias económicas y simbolismo en el uso de las remesas de la migración ecuatoriana en España. Tesis Doctoral, Tarragona, Universitat Rovira i Virgili.

SAYAD, A. (1999): La doublé absence. Des illusions de l'émigré aux souffrances de l'immigré. Paris, Éditions du Seuil

Solé, C., Parella, S. Y CavalCanti, L. (2007): Los vínculos económicos y familiares transnacionales. Los inmigrantes ecuatorianos y peruanos en España. Madrid, Fundación BBVA.

SUÁREZ, L. (2007a): «Identitat, territori i ciutadanies en el camp migratori transnacional». Revista d'Etnologia de Catalunya, n. ${ }^{\circ} 30, \mathrm{pp} .45-69$.

- (2007b) «La perspectiva transnacional en los estudios migratorios. Génesis, derroteros y surcos metodológicos». Actas del V Congreso sobre la Inmigración en España. Migraciones y Desarrollo Humano, Valencia: CEIM y Universitat de Valencia. Disponible en http://www.adeit.uv.es/inmigracion2007/index.php y publicado en CD- Rom. 
WAMSLEY, E. (2001): «Transformando los pueblos: la migración internacional y el impacto social a nivel comunitario». Ecuador Debate, n. ${ }^{\circ}$ 54, pp. 155-174.

\section{RESUMEN}

En los últimos años, la perspectiva transnacional se ha convertido en el paradigma dominante dentro de los estudios sobre migraciones. Dentro de este marco teórico han sido numerosas las temáticas que se han abordado, siendo los estudios sobre remesas uno de los aspectos que ha suscitado un interés mayor dada su creciente magnitud en múltiples países.

La mayor parte de los trabajos realizados sobre envíos de dinero han abordado la vertiente económica del fenómeno, predominando frecuentemente en ellos un enfoque macroeconómico dominado por datos estadísticos que busca estimar la magnitud de las remesas, así como analizar el fin al que éstas se destinan. Sin embargo, son escasos los trabajos que han abordado el estudio de las remesas en el marco más amplio de las estrategias económicas seguidas por los migrantes, y su relación con el espacio hacia el que los migrantes proyectan su movilidad social.

Para realizar una aproximación a las remesas desde esta perspectiva, en este artículo se presenta como aportación metodológica, un instrumento para recoger información denominado «migragrama», desarrollado especialmente para explorar las relaciones entre el proyecto migratorio, las estrategias económicas seguidas y el envío de remesas realizadas.

\section{PALABRAS CLAVE}

Transnacionalismo, remesas, técnica metodológica, estrategias económicas, movilidad social.

\section{ABSTRACT}

In the last years, the transnational perspective has become the dominant paradigm in studies on migrations. Within this theoretical framework, numerous topics have been dealt with, being studies about remittances one of the topics which have aroused most interesting, due to its increasing magnitude in many countries.

Most of the researches carried out on the subject of money transfers has approached the economic side of the phenomenon, which seeks to estimate the magnitude of the remittances, as well as to analyze the purpose with which they are made. However, few works have approached the study of remittances in 
the wider frame of the economic strategies followed by migrants and their connexions with the place where migrants are planning their social mobility.

Doing an approach to the remittance from this perspective, it is presented in this paper an instrument to collect information developed in this called «migragrama» which was especially developed for exploring the relations among migratory project, economic strategies and sending of remittances.

\section{KEY WORDS}

Transnationalism, remittances, methodological technical, economic strategies, social mobility. 